\title{
A Routing Protocol for Wireless Sensor Networks with Congestion Control
}

\author{
Qi Li ${ }^{1}$, Zongwu Ke ${ }^{2 *}$, Duanfeng Xia ${ }^{3}$, Yuxia Sun ${ }^{4}$ \\ School of Computer Science and Technology, Hubei Normal University, Huangshi Hubei, China \\ Email: hbxfliqi2012@126.com, kezongwu@126.com, aimixia@163.com,33311454@qq.com
}

Received May, 2013

\begin{abstract}
This paper proposed a novel RED protocol which takes the node's energy into account depending on the length of the data packet. It also proposed a routing protocol for wireless sensor networks with congestion control which imitates the ant colony foraging behavior. Sensor nodes choose routings according to the pheromone density. The experiment result shows that the algorithm balances the energy consumption of each node. It mitigated congestion effectively with the proposed routing protocol.
\end{abstract}

Keywords: Wireless Sensor Network; Routing; Congestion Control

\section{Introduction}

Wireless sensor network is one of the key technologies in IoT. Many applications based on wireless sensor networks usually require real-time multimedia data [1], such as health-care system, target tracking, etc. In wireless sensor network, sensor node usually has small memory capacity, and the buffer resources used for routing are relatively few, but real-time multimedia has a large amount of data, a large number of multimedia data transfer to sink node via multi-hops, it's easy to generate congestion. Transmission delay generated by network congestion can't meet real-time requirement of multimedia data. As we know, the sensor node is usually powered by battery, node in congestion zone consumes huge energy, it would lead some nodes energy exhausted in a local network area, it also reduces the lifetime of the network [2].

Form the above discussion, the routing protocol of wireless sensor networks with large data traffic not only needs to find an optimized path, but also needs congestion avoidance and detection.

This paper studies routing protocol with congestion control in wireless sensor networks.

The remainder of this paper is organized as follows. Section 2 gives a brief overview of the related work. Section 3 presents an improved random early detection model. Section 4 presents a routing protocol for wireless sensor networks with congestion control. Section 5 discusses the simulation model and the experimental re-

${ }^{*}$ Corresponding author. sults. Section 6 concludes this paper.

\section{Related Work}

Congestion control is a strategy that is used to avoid network congestion and mitigate congestion. Wireless sensor networks are easy to generate congestion because of its constrained resource. Once network generates congestion, it would bring a lot of resources consumption. So it is necessary to use congestion control to avoid congestion in wireless sensor networks. Congestion control in wireless sensor networks usually have two types, end-to-end and hop-by-hop congestion control [3]. Congestion control includes congestion detection and congestion avoidance.

Congestion detection often relies on the buffer's occupation [4-7].Mohammad proposed the distinction service and congestion control strategy which is a kind of end-to-end type, based on priority rate in wireless sensor network multimedia, and used a strategy to avoid congestion which is similar to RED [6]. Bret Hull proposed a congestion control strategy which uses a combination of hop-by-hop flow control, rate limiting source traffic and a prioritized medium access control protocol [3]. Its basic idea is to set a congestion bit through different congestion detection methods. It will broadcast congestion information to the neighbor node if the node generates congestion. Its congestion detection method is to judge whether it will generate congestion using prediction method. This idea doesn't take different types of sensing data in sensor networks into account. When the size of the data packet is very small, the congestion node actu- 
ally can forward the packet. Omar Banimelhem proposed a wireless sensor networks' multi-path routing protocol with congestion control based on grid [7]. Its method to reduce congestion is monitoring the buffer through the master node. If a buffer occupies more than a threshold value, it broadcasts congestion information to the neighbor node. And choose two master nodes in grid, master node and secondary master node. When the master node produces congestion, it is a duty for secondary master node to route in order to reduce congestion.

\section{Improved Random Early Detection Model}

Random Early Detection (RED) is a congestion-avoidance mechanism. RED also known as random early drop, it will drop the packets with statistical probabilities based on the average queue size [8].

There are different data types in wireless sensor networks, and the length of every data packet is quite different from each other. Many sensed information, such as the temperature whose length of data packet only a few bytes. But the length of the multimedia data packets may occupy several $\mathrm{K}$ bytes, the RED protocol which is designed by the main idea of simple queue length can't meet the requirements of wireless sensor networks.

So, we design a novel random early detection model which is decided by the size of data packets.

First we give a method of calculating the average data length in the present buffer with the weighted average method.

$$
\begin{aligned}
\text { AvgDataLen }= & (1-\text { Weight }) \times \text { AvgDataLen } \\
& + \text { Weight } \times \text { SampleDataLen }
\end{aligned}
$$

Where SampleDataLen is the data length which is detected in the present buffer, AvgDataLen is the weighted average length. The current sample value is sampling when a package is completely received.

The drop probability function of RED is definite as follows:

Suppose the size of the sensor node's buffer is max buffer size, set two thresholds to control the drop probability.

If AvgDataLen $\leq$ MinBufferThreshold , the sensor node accepts data directly, that is, $p=0$.

$$
\text { If }
$$

MinBufferThreshold $<$ AvgDataLen $\leq$ MaxBufferThreshold, the sensor node drops following data packet with probability $p$.

If AvgDataLen>MaxBufferThreshold, the sensor node drops following data packet with probability $p=1$.

That is, if average data length is smaller than MinBufferThreshold, it doesn't take any measure; if average data length is between MaxBufferThreshold and MinBufferThreshold, it drops the following data packets with probability $p$; if average data length is longer than
MinBufferThreshold, it drops the packet directly.

The sensor nodes in wireless sensor networks are usually powered by battery. If the sensor node is used excessively, it will bring energy exhaustion. The network is easy to become invalid. It will lead to reduce lifetime in wireless sensor networks. When the node's residual energy is low, it will drop data actively. It will inform the sender to choose other nodes with high energy to transmit data.

It gets the abandon rate $P$ with the energy factor $e$ based on the traditional RED model.

$$
\begin{aligned}
& \text { TempP } \\
& =\text { Maxp } \times \frac{\text { AvgDataLen-MinBufferThreshold }}{\text { MaxBufferThreshold-MinBufferThreshold }} \times e \\
& P=\frac{\text { TempP }}{1-\text { Datalen } \times \text { TempP }}
\end{aligned}
$$

DataLen is the size of the data packet coming in the node which is not discarded, while the size of average data between the two threshold.

$$
\text { Maxp }=1 / 32=0.03125
$$

The energy model uses hierarchical mechanism:

$$
x \in[0,1], e=2^{6-\lceil 6 x\rceil}
$$

$x$ represents the ratio of the residual energy and the maximum energy

Assume the MinBufferThreshold is $500 \mathrm{~KB}$ and the MaxBufferThreshold is $1000 \mathrm{~KB}$, the drop probability is shown as Figure 1:

\section{A Routing Protocol for Wireless Sensor Network with Congestion}

Routing protocols in wireless sensor networks have been researched widely. Generally, routing protocols are divided into table-driven routing protocols and on-demand

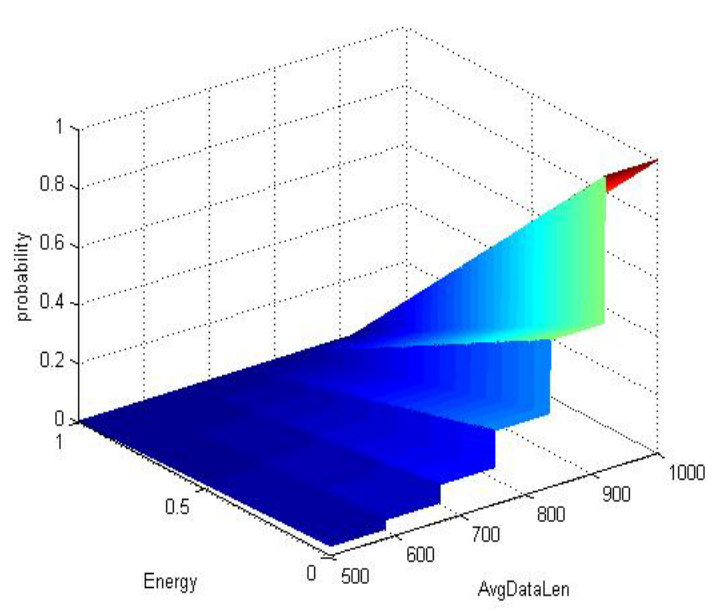

Figure 1. Drop probability with energy. 
routing protocols. General routing protocols in wireless sensor networks don't take congestion control into account.

Some routing protocols using ant colony algorithm have been studied [9-11]. This paper proposes a novel routing protocol of wireless sensor network which imitate ant colony foraging behavior. Ants usually leave pheromone on the path when looking for food and they always follow the path with high pheromone density in order to find an ideal path. To imitate the ants' habit, we maintain a routing table in each node. All neighbor nodes' pheromone density $Q_{n}$ of a node store in this routing table. When a node prepares to send data, it chooses the next hop with probability $P_{n}$ shown as follows:

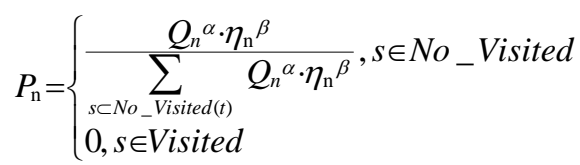

where, $\eta_{\mathrm{n}}$ is inspiration function, it represents the ratio of The residual energy and the total energy, that is

$$
E_{r} / E_{t}
$$

$\alpha$ is the important degree factor of pheromone, indicating the importance of pheromone in the process of choosing the next hop.

$\beta$ is the important degree factor of inspiration function, indicating the importance of inspiration function in the process of choosing the next hop. No_Visited tells node set that the current node didn't visit. Accordingly, Visited tells node set that the current node has visited.

Suppose that there is only one sink node in wireless sensor networks. If a node is in the condition of initialization, the neighbor nodes' pheromone densities in its routing table are all zero.

When the node prepares to send data, it needs to initialize its routing table, initialize method as Figure 2:

Step 1: The source node send a path-finding packet to all neighbor nodes, the path-finding packet contains source node identification.

Step 2: If the next hop has completed initialization, find the next hop by $P_{n}$, and record this path in the path-finding packet; Else Initialize this node

Step 3: If it is not a sink Go to Step 2; Else Send back all paths' pheromone density to source node

Figure 2. The algorithm of initializing the routing table.
Sink node calculates source's neighbor nodes' pheromone density in its routing table according to the hop count on each path, the formula is as follows:

$$
Q_{n}=\left(1-\frac{\operatorname{hopcount}(n)}{\sum \operatorname{hopcount}(n)}\right) \times Q
$$

where hopcount(n) represents the hops on each path, $Q$ is a constant number.

If a neighbor node doesn't appear in the routing table sent back by sink node, it shows that this node can't reach sink node, we set its pheromone density zero.

The routing table updating strategy is defined as follows:

A protocol is proposed to transmit data hop-by-hop which is similar to stop-wait-protocol in this paper.

A volatile factor $\rho$ is used to change the pheromone density, pheromone density on current path volatile with $\rho$ and the pheromone density will be updated with $\Delta \tau_{t}$ after finding a path, that is,

$$
Q_{i}=(1-\rho) Q_{i}+\Delta \tau_{t}, 0<\rho<1
$$

where $Q_{i}$ represents the pheromone density of the node i.

It sets a timeout timer in this protocol. When a node sends a data packet, it waits for the confirm message from the next hop. If a node receives a confirm message from the next hop, it indicates that the node sends data packet successfully. It updates the nodes' pheromone densities in the current node routing table with the following method.

$$
\Delta \tau_{t}=\left\{\begin{array}{l}
Q, t=i \\
0, \text { others }
\end{array}\right.
$$

If it times out, reduce the pheromone density with the following method:

$$
Q_{i}=\frac{Q_{i}}{2^{k}}
$$

where $k$ presents the number of timeout. This method can reduce the congestion node's energy sharply and it can guarantee the validity of the routing.

\section{Experimental Results}

In this paper, it simulates this routing algorithm using the topology of network shown in Figure 3. In the topology, there are three sensor nodes $S_{1}, S_{2}, S_{3}$, three routers $r_{1}, r_{2}, r_{3}$ and a sink node. A sensor node $S_{i}(i=1,2,3)$ sends data packets to the sink node via router $r_{i}(i=1,2,3)$. The network topology is shown in Figure 3.

In the experiment, the sensor node sends data with the speed of 100 bytes per second. Set $500 \mathrm{~KB}$ as the MinBufferThreshold and set $1000 \mathrm{~KB}$ as the MaxBufferThreshold. Assume that the initial energy of $r_{1}, r_{2}, r_{3}$ are $E_{r 1}=9, E_{r 2}=12, E_{r 3}=10$. 
In the first experiment, it uses both traditional RED protocol and the proposed RED protocol to watch on the condition of drop probability. When taking traditional RED protocol, three nodes all select the router $r_{2}$ to send data packet. The drop probability increases to maximum value and keeps it. In the proposed protocol, three nodes all select the router $r_{2}$ at first. The drop probability in $r_{2}$ will increase if there is congestion. Then, dropped data will find other nodes to send .The energy in the proposed RED protocol uses equation.(4)..

The first experimental result is shown in Figure 4, G1 is the drop probability curve by the algorithm of RED, and the drop probability rises until the maximum and keeps it. When it takes the proposed protocol, the node drop data with some probability if there is congestion. The dropped data selects some other node, so the drop probability of the current node reaches a certain value at first, and then it decreases tending to a small probability.

In the second experiment, it uses traditional routing protocol and the proposed routing protocol to watch on the energy consumption condition.

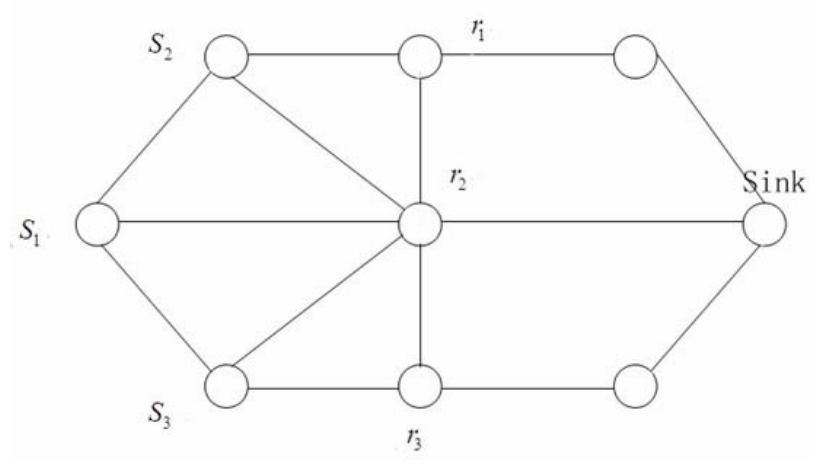

Figure 3. The topology of network.

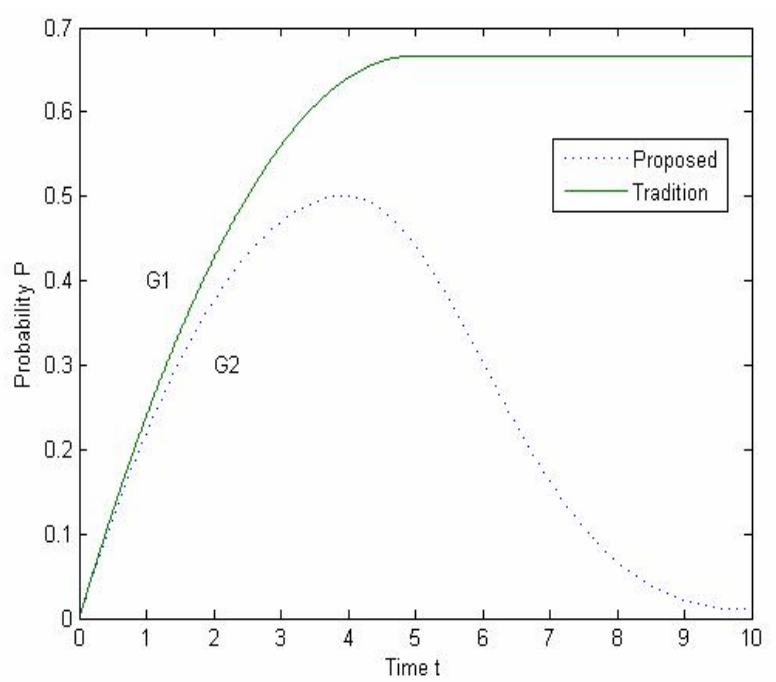

Figure 4. Drop probability in traditional RED vs. proposed RED.

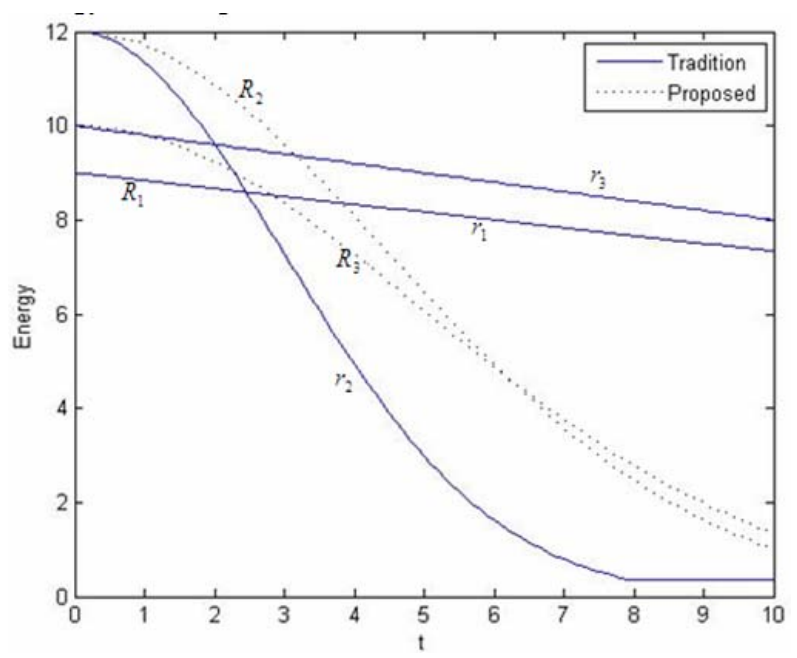

Figure 5. Energy consumption in proposed routing protocol vs. traditional.

When taking the traditional routing protocol, three nodes $S_{1}, S_{2}, S_{3}$ all choose the router $r_{2}$, so the energy of $r_{1}$ nearly doesn't change and the energy of $r_{3}$ nearly doesn't change either. The energy of $r_{2}$ changes as the curve $r_{2}$ shown in Figure 5. The energy of the router $r_{2}$ reduces sharply because three nodes all choose the router $r_{2}$. When the energy of the router $r_{2}$ reaches a certain value $E_{0}$, it keeps this value, the energy of the router $r_{2}$ tends to be $E_{0}$ When taking the proposed routing protocol, $S_{1}, S_{2}, S_{3}$ choose routes $r_{2}$ at first. Once it generates congestion in router $r_{2}$, router $r_{2}$ will drop data with a certain probability $p_{0}$, and the dropped data will choose the router $r_{3}$. So the router $r_{3}$ could consume some energy. The energy of $S_{2}$ changes as the curve $R_{2}$ in Figure 5, and the energy of $S_{3}$ changes as the curve $R_{3}$ in Figure 5.

From the analysis of the drop probability and energy above, using the improved model could reduce the drop probability and reduce congestion effectively. Meanwhile, the energy state of the node which generated congestion before has an improvement. All of the data don't choose a unique node, so that it can reduce the energy consumption.

\section{Conclusions}

This paper proposed a routing protocol of wireless sensor networks with congestion control. The routing algorithm simulates the ant colony foraging behavior.

The RED protocol takes the balance use of energy into account. The experiment shows that the protocol could avoid congestion and mitigate congestion effectively. Meanwhile, it could prolong the network's lifetime.

The following research work is to compare this protocol with general wireless sensor network routing protocol by simulation and study the characters of the protocol. 


\section{Acknowledgements}

The work was supported by Hubei provincial universities cooperation projects in China (Grant NO: CXY2009B031), and the plan for scientific and technological innovation team of excellent young and middle-aged in institute of high learning of Hubei Province in China (Grant No:T200806).

\section{REFERENCES}

[1] I. F. Akyildiz, T. Melodia and K. R. Chowdhury, "A Survey on Wireless Multimedia Sensor Networks," Computer Networks, Vol. 51, No. 4, 2007, pp. 921-960. doi:10.1016/j.comnet.2006.10.002

[2] I. Dietrich and F. Dressler, "On the Lifetime of Wireless Sensor Networks," ACM Transactions on Sensor Networks, Vol. 5, No. 1, 2009, pp. 1-38. doi:10.1145/1464420.1464425

[3] B. Hull, K. Jamieson and H. Balakrishnan, "Mitigating Congestion in Wireless Sensor Networks," Proceedings of ACM Sensys'04, Baltimore, MD, November 3-5, 2004.

[4] Md. A. Razzaque and C. S. Hong, "Congestion Detection and Control Algorithms for Multipath Data Forwarding in Sensor Networks," International Conference on Advanced Communication Technology, February 15-18, 2009, Gangwon-Do, Korea, pp. 651-653.

[5] M. Z. Ahmad and D. Turgut, "Congestion Avoidance and Fairness in Wireless Sensor Networks,” Global Tele- communication Conference, USA, 2008 November 30-December 4, New Orleans.

[6] Y. M. Hossein and D. A. Adjeroh, "Priority-based Rate Control for Service Differentiation and Congestion Control in Wireless Multimedia Sensor Networks," Computer Networks, Vol. 53, No. 11, 2009, pp. 1798-1811. doi:10.1016/j.comnet.2009.02.011

[7] O. Banimelhem and S. Khasawneh, "Grid-based Multipath with Congestion Avoidance Routing Potocol in Wireless Sensor Networks," Ad Hoc Netw, 2012, doi:10.1016/ j.adhoc.2012.03.015

[8] Floyd Sally and Jacobson Van, "Random Early Detection (RED) Gateways for Congestion Avoidance,” IEEE/ACM Transactions on Networking, Vol. 1, No. 4, 1993, pp. 397-413. doi:10.1109/90.251892

[9] W.-H. Liao, Y. C. Kao and C.-M. Fan, “Data Aggregation in Wireless Sensor Networks Using Ant Colony Algorithm," Journal of Network and Computer Applications, Vol. 31, No. 4, 2008, pp. 387-401. doi:10.1016/j.jnca.2008.02.006

[10] J.-H. Ho, H.-C. Shih, B.-Y. Liao and S.-C. Chu, “A Ladder Diffusion Algorithm Using Ant Colony Optimization for Wireless Sensor Networks," Information Sciences, Vol. 192, 2012, pp. 204-212. doi:10.1016/j.ins.2011.03.013

[11] W.-H. Liao, Y. C. Kao and R.-T. Wu, “Ant Colony Optimization Based Sensor Deployment Protocol for Wireless Sensor Networks,” Expert Systems with Applications, Vol. 38, No. 6, 2011, pp. 6599-6605. doi:10.1016/j.eswa.2010.11.079 